This is a postprint version of the following published document:

Prior, E.; Dios, C. de; Ortsiefer, M.; Meissner, P. and Acedo, P. (2015). Understanding VCSEL-based gain switching optical frequency combs: experimental study of polarization dynamics. Journal of Lightwave Technology, 33 (22), pp. 4572-4579

DOI: $10.1109 /$ JLT.2015.2476956

(C) 2015 IEEE. Personal use of this material is permitted. Permission from IEEE must be obtained for all other uses, in any current or future media, including reprinting/republishing this material for advertising or promotional purposes, creating new collective works, for resale or redistribution to servers or lists, or reuse of any copyrighted component of this work in other works. 


\title{
Understanding VCSEL-Based Gain Switching Optical Frequency Combs: Experimental Study of Polarization Dynamics
}

\author{
Estefanía Prior, Cristina De Dios, Markus Ortsiefer, Peter Meissner, Member, IEEE, \\ and Pablo Acedo, Member, IEEE
}

\begin{abstract}
In this paper, we carry out an experimental study on the polarization properties of a vertical-cavity surface-emitting laser (VCSEL) working under gain switching (GS) regime and the characteristics of the resulting optical frequency comb signal. We have observed that each of the two polarization modes presented in the VCSEL continuous wave emission spectrum generate a separate optical frequency comb (OFC) whose modes are phase correlated thanks to the GS regime. We study how these combs associated with the main and orthogonal polarization modes, respectively, vary depending on the input parameters to the VCSEL (bias current and radio-frequency power and frequency). The correlation between both OFCs in the best operation point as defined by the OFC characteristics is also evaluated. Therefore, this study demonstrates that two orthogonally polarized combs are generated that exhibit a high correlation between each other that combine to produce a wider overall optical comb. Hence, we can predict the feasibility of dual-polarization VCSEL-based OFC generators in the few gigahertz repetition frequency rates, with highly correlated modes and continuously tunable distance between them, in a compact and energy- and cost-efficient system that can find application in ultrafast laser dynamics studies and or in polarization-division multiplexing optical communications.
\end{abstract}

Index Terms-Energy efficiency, gain switching (GS), laser diodes (LDs), optical frequency comb generator (OFCG), orthogonal mode, polarization switching, vertical-cavity surface-emitting laser (VCSEL).

\section{INTRODUCTION}

$\mathbf{O}$ PTICAL frequency comb generators (OFCG) are versatile systems that find application in many disciplines like spectroscopy [1], optical communications [2], THz generation [3], optical arbitrary waveform generation, metrology, or microwave photonic [4]. Among OFCGs, those based on cost of

The work of E. Prior was supported by the Spanish Ministry of Economy and Competition under the Retos-Colaboración Program, RTC-2014-2661-7 in collaboration with Luz WaveLabs S.L. This work was supported by the Spanish Ministry of Economy and Competitiveness under RTC-2014-2661-7 and TEC-2014-52147-R Grants.

E. Prior, C. De Dios, and P. Acedo are with the Electronics Technology Department, Universidad Carlos III de Madrid, Leganés, Madrid 28911, Spain (e-mail: eprior@ing.uc3m.es; cdios@ing.uc3m.es; pag@ing.uc3m.es).

M. Ortsiefer is with Vertilas GmbH, Munich 85748, Germany (e-mail: ortsiefer@vertilas.com).

P. Meissner is with the Technische Universität Darmstadt, Darmstadt 64289, Germany (e-mail: meissner@imp.tu-darmstadt.de).

Color versions of one or more of the figures in this paper are available online at http://ieeexplore.ieee.org. the shelf (COTS) laser diodes (LDs) are interesting systems since they offer compactness and cost efficiency.

There are numerous diode laser technologies. One of the most interesting ones is vertical-cavity surface-emitting laser (VCSEL) devices [2], [5], [6]. Previous studies have demonstrated that VCSEL diode lasers under gain switching (GS) regime [7] produce record combs in terms of energy efficiency and mode coherence. The interest of such combs could be extended if wider optical spans could be achieved while maintaining its high quality capabilities. In this sense, the use of external comb extension techniques, like electro-optical modulators could be detrimental as they are costlier components and have higher energy needs [8]. A deeper understanding of the dynamics of VCSEL under GS to generate optical combs could help us comprehend how to obtain wider combs using a single stage OFCG. This is the main focus of this experimental work, where we will center our attention in the polarization dynamics of VCSEL devices under GS operation to produce optical frequency combs. VCSELs are typically considered to be single-longitudinal-mode devices. However, their emission consists of two linearly polarized modes with orthogonal polarizations that, due to anisotropies in the materials, emit at different wavelengths [2], [9]. Manufacturers have developed effective techniques to minimize this duality and present devices that exhibit a side mode suppression ratio above $30 \mathrm{~dB}$, what makes this device purely monomode for many applications. However, we believe that this suppressed mode can play an important role in VCSEL-based OFCG.

In this work we present new results on our study of VCSELbased GS-OFCGs. We evaluate the dynamic behaviour of these two orthogonal modes of polarization present in a VCSEL under GS and how this affects the overall OFCG. We have observed that each polarization mode generates a separate optical comb and we have analysed the evolution of each of them with the input parameters that determine the GS operation of a VCSEL device (bias and RF modulation). On the other hand, and more importantly, we have seen how these orthogonally polarized combs that appear are related in phase and collaborate to produce a wider overall total comb. This is remarkable if we take into account that the two modes present high anticorrelation if no modulation is applied as it is stated in [10], [11]. Therefore, this will imply that the phase correlation is caused by the GS regime. This result suggests that an enhancement of the emission of the secondary mode, through fabrication or external injection techniques, would help overcome one of the disadvantages of 


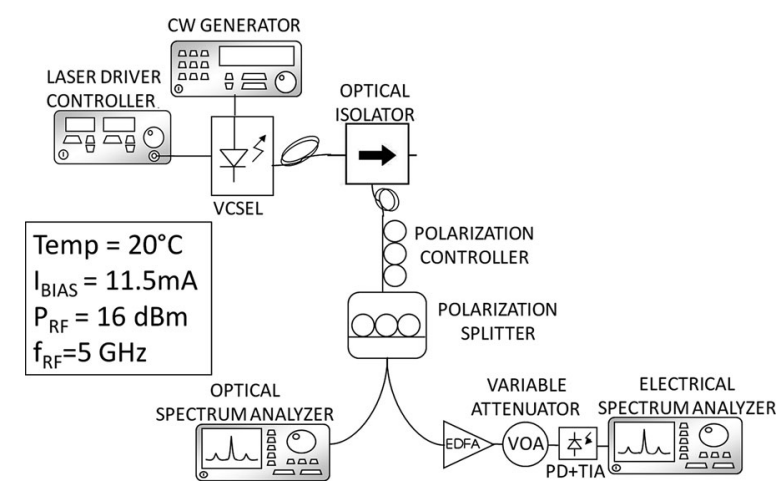

Fig. 1. Experimental set-up. The comb is generated inside the VCSEL and both polarization components are split and presented at each output of the PS. The optical and electrical spectra are measured in the OSA and ESA respectively. See text for details.

this OFCG, which is the limited optical span, if compared to more complicated sources. Another advantage is that the availability of two coherent combs with orthogonal polarizations can find applications in ultrafast laser dynamics studies [12] or in polarization-division multiplexing optical communications [13].

\section{EXPERIMENTAL STUDY}

Fig. 1 details the experimental set-up we have used for the present work. The VCSEL diode laser device under study is a state of the technology device (VERTILAS VL-1550-8G-P2$\mathrm{H} 4)$ provided together with a specific board for radiofrequency (RF) operation within the laser bandwidth. The comb is generated inside the VCSEL cavity using the non-linear GS regime. Under GS operation, a large RF signal is injected in the laser cavity forcing its pulsed operation. Therefore an optical comb is created in the frequency domain. In our experiments, we will mainly use the VCSEL temperature stabilized at $20{ }^{\circ} \mathrm{C}$ with a bias current of $11.5 \mathrm{~mA}$ and an input RF signal of $16 \mathrm{dBm}$ at $5 \mathrm{GHz}$ (Fig. 1). Any change on these parameters will be noted in the text. This is the working point where we found the best optical comb characteristics, as it will be explained in the next section.

After the VCSEL, an optical isolator is placed to avoid any optical feedback. The output of the VCSEL is composed of two modes orthogonally polarized that are split with a polarization controller (PC) and a fiber polarization splitter (PS) from Thorlabs (PBC1550PM-APC). We will measure the optical spectra in an optical spectrum analyzer (OSA) with $0.002 \mathrm{~nm}$ resolution and the electrical spectra in an electrical spectrum analyzer (ESA) using an ultrafast $50 \mathrm{GHz}$ photodetector. Before the photodetector, an erbium-doped fiber amplifier and a variable optical attenuator are used to equalize the carrier power when needed.

In this work, $P$ or total signal is the total output power of the VCSEL, including the main mode $P x$, with parallel polarization, and the suppressed side mode $P y$, with orthogonal polarization.

In Fig. 2 we show the PI curves of the two modes with orthogonal polarizations: $P x$ and $P y$ in $\mathrm{dBm}$. These optical powers have been measured placing a powermeter after each output of

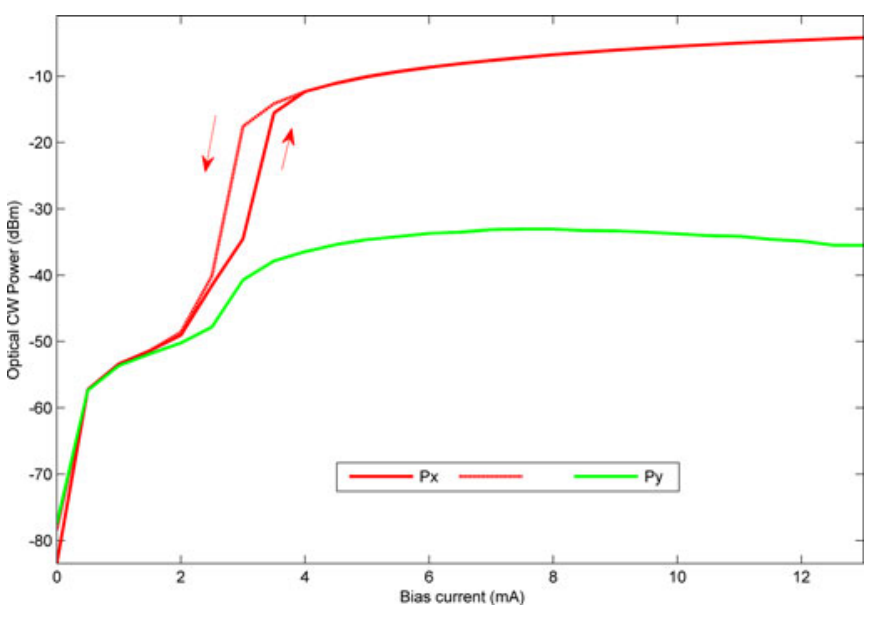

Fig. 2. $\quad$ PI Curves for $P x$ and $P y$. The main polarization mode, $P x$, emits most of the power at the output of the VCSEL and the orthogonal mode power is $>40 \mathrm{~dB}$ below the main mode.

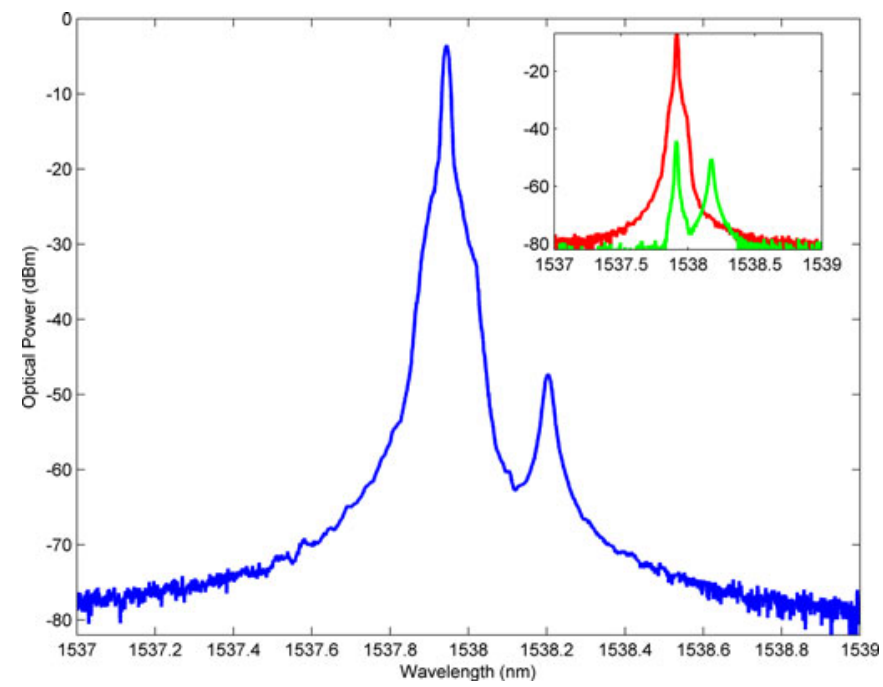

Fig. 3. CW emission spectra for $P, P x$ and $P y$. The dominant main mode in $P$ at $1537.95 \mathrm{~nm}$ is more than $40 \mathrm{~dB}$ above the residual orthogonal mode shown at $1538.20 \mathrm{~nm}$. The birefringence of this VCSEL is $31.21 \mathrm{GHz}$. Both outputs of the PS selecting $P x$ (red line) and $P y$ (green line) respectively are presented in the figure inset.

the PS and tuning carefully the PC to maximize the contribution of the mode of interest ( $P x$ or $P y)$.

Below and around threshold $\left(I_{\mathrm{BIAS}}=2.5 \mathrm{~mA}\right)$ both components have similar power levels. Above threshold, $P x$ and $P y$ are always present but the main mode, $P x$, emits most of the total power $(P x / P y>40 \mathrm{~dB})$. However, the orthogonal mode shows an increase in emission $>10 \mathrm{~dB}$ when crossing the threshold, what means it can be considered a lasing mode. We also observe hysteresis around threshold in the PI curves in both modes that shows a parallel evolution with respect to each other. From these results we conclude that the device under study exhibits a stable emission without polarization switching bistable regions. Following the classification of [14], this VCSEL is type c because both modes lase at the same time. 

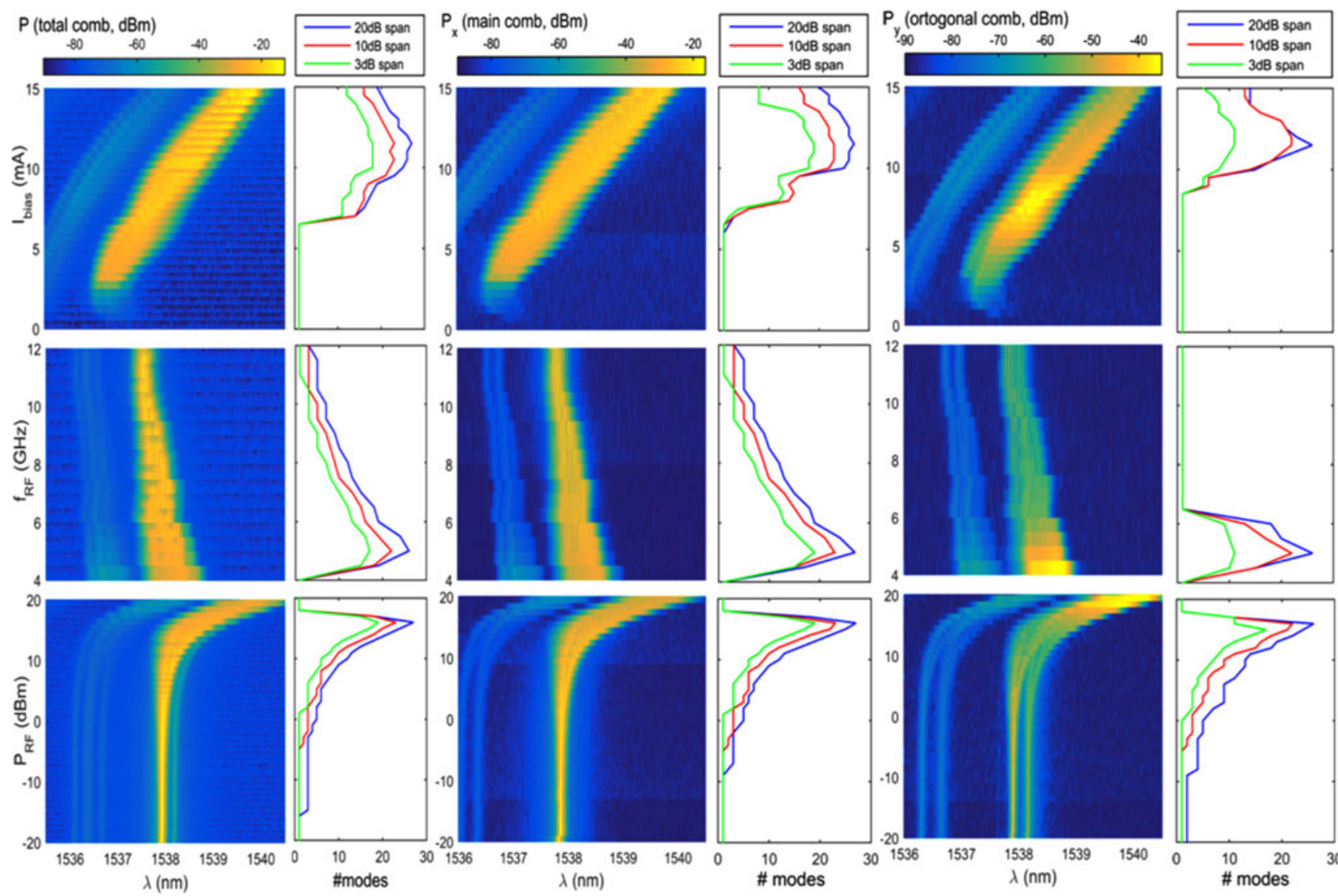

Fig. 4. Left: total comb (P-OFCG): maps with its evolution with bias current, RF frequency and RF power respectively and curves detailing the number of modes at 3,10, and $20 \mathrm{~dB}$ spans. Center: Main comb ( $P x$-OFCG) maps with its evolution with bias current, RF frequency and RF power respectively, and curves detailing the number of modes. Right: Orthogonal comb (Py-OFCG) maps with its evolution with bias current, RF frequency and RF power respectively, and curves detailing the number of modes. The broadest comb for the three signals under study $-P, P x$ and $P y-\mathrm{occurs}$ at $\mathrm{T}=20{ }^{\circ} \mathrm{C}, I_{\mathrm{bias}}=11.5 \mathrm{~mA}, f_{\mathrm{RF}}=5$ $\mathrm{GHz}$ and $P_{\mathrm{RF}}=16 \mathrm{dBm}$.

In Fig. 3 we can see that at $11.5 \mathrm{~mA}$ the VCSEL presents a clear main $P x$ mode at $1537.95 \mathrm{~nm}$ with $-4.2 \mathrm{dBm}$ peak power and an orthogonal $P y$ mode at $1538.20 \mathrm{~nm}$ with $-47.3 \mathrm{dBm}$. Then, there is a $43.1 \mathrm{~dB}$ power ratio between the $P x$ and $P y$ signals and the frequency difference of the modes with orthogonal state of polarisation due to the birefringence of the laser amounts to $31.21 \mathrm{GHz}$, what is consistent with the manufacturer's specifications.

The signals at each output of the PS, $P x$ and $P y$ are also shown (inset Fig. 3). When the $P y$ signal is selected (light with orthogonal polarization), a residual part of the main $P x$ mode is still detectable even if the PS component reduces its contribution more than $30 \mathrm{~dB}$. Using several PS elements in cascade to improve the polarization selectivity gives the same result as in Fig. 3, so it does not improve the selectivity as, at the output of the first component the polarization in both wavelengths is now the same. Then, from now on, Px signal is the light at the output of the VCSEL with parallel polarization, which coincides with the main lasing mode and Py signal is constituted by all the light with orthogonal polarization, including the secondary mode and a residual part (minimized but still detectable) of the light of the main mode.

\section{A. Optical Frequency Comb Generation and Characteristics}

Under GS operation, the VCSEL generates different optical combs depending on the amount of RF power injected to modulate the laser $\left(P_{\mathrm{RF}}\right)$, its frequency $\left(f_{\mathrm{RF}}\right)$, and the bias current applied ( $I_{\text {BIAS }}$ ). In Fig. 4 we depict the maps detailing the evolution of the total comb of the VCSEL $P$-OFCG (left), the $P x$ OFCG (centre) and the $P y$-OFCG (right) when each of these parameters change with respect to the reference values mentioned above. For each comb we show three maps and three figures. The figures correspond to the number of modes exhibited by the comb under study when each of the parameters controlling the $\mathrm{GS}$ regime changes. The number of modes is evaluated considering $3 \mathrm{~dB}, 10 \mathrm{~dB}$ and $20 \mathrm{~dB}$ optical bandwidths. For applications such as $\mathrm{THz}$ photonic generation, the useful optical span can be defined up to $20 \mathrm{~dB}$ [15]. For optical communications, the flatness of the comb is more critical and the useful modes lie within a 3 or $10 \mathrm{~dB}$ bandwidth [16]. This is why we have evaluated the comb span using several metrics.

In Fig. 4(left) we observe that the total $P$-OFCG redshifts when $I_{\text {BIAS }}$ increases. The broadest comb is obtained at $11.5 \mathrm{~mA}$. The RF frequency affects the comb differently and its width decreases with the frequency when we try to drive 


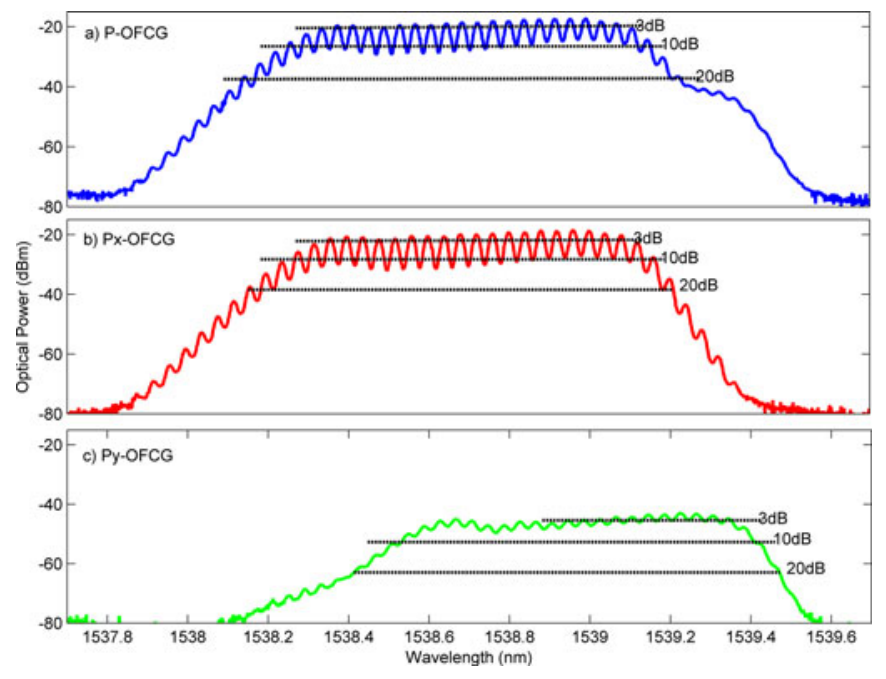

Fig. 5. Best point combs obtained at $20^{\circ} \mathrm{C}, 11.5 \mathrm{~mA}, 5 \mathrm{GHz}, 16 \mathrm{dBm}$. (a) The upper line corresponds to the total output of the VCSEL, P-OFCG, with both polarization modes included. (b) The middle line is the comb $P x$-OFCG, generated only with the parallel polarization signal. (c) The lower line is the comb $P y$-OFCG which has the orthogonal polarization. The combs $P$-OFCG and $P$-OFCG have 27 lines in the $20 \mathrm{~dB}$ span which corresponds to $135 \mathrm{GHz}$ bandwidth. The comb $P y$-OFCG has one mode less, 26 in total, so the bandwidth in this case is $130 \mathrm{GHz}$

the device above the modulation bandwidth of the laser (around $5 \mathrm{GHz}$ ), what weakens the GS regime. The broadest comb in this case, appears at $5 \mathrm{GHz}$. Finally, the comb evolution with the RF power shows how the comb broadens with the injection of power, as the GS regime accentuates. However, above $17 \mathrm{dBm}$, the device enters into period doubling operation and the total comb does not broaden any further. We are not interested in this nonlinear regime of the VCSEL as our focus now is a tunable comb with a directly controllable repetition frequency equal to $f_{\mathrm{RF}}$. Therefore, the broadest comb takes place for $11.5 \mathrm{~mA}$, $5 \mathrm{GHz}$ and $16 \mathrm{dBm}$. In Fig. 4(left) (upper figure, blue line) we show this comb, which has a total of 27 lines which corresponds to $135 \mathrm{GHz}$ considering $20 \mathrm{~dB}$ span.

The same evolution study has been done for $P x$ and $P y$. It is very interesting to note that the characteristics of these subcombs are similar to the ones presented by the total $P$-comb: we observe the red-shift associated to the increase of the bias current and the opposite happens with the RF frequency due to the modulation bandwidth of the VCSEL. For both $P x$ and $P y$, the optimum comb in terms of span occurs at $I_{\mathrm{BIAS}}=11.5 \mathrm{~mA}$, $f_{\mathrm{RF}}=5 \mathrm{GHz}$ and $P_{\mathrm{RF}}=16 \mathrm{dBm}$. Varying these parameters will imply either a narrower comb or the appearance of period doubling operation. Of course, the orthogonal mode exhibits an optical comb with lower optical power and a slightly higher central wavelength. However, the power ratio between the $P x$ and $P y$ optical combs is now only $26 \mathrm{~dB}$, when it reached $43.1 \mathrm{~dB}$ under $\mathrm{CW}$ operation. This is one of the most important results of our study as the GS regime enhances the power associated to the orthogonally polarized signal $P y$.

In Fig. 5 we analyse the optical combs generated in the best operation point in terms of optical span and flatness described before $\left(T=20^{\circ} \mathrm{C}, I_{\mathrm{BIAS}}=11.5 \mathrm{~mA}, f_{\mathrm{RF}}=5 \mathrm{GHz}, P_{\mathrm{RF}}=16\right.$
TABLE I

COMPARISON TOTAL MAIN AND ORTHOGONAL OFCG

\begin{tabular}{lccc}
\hline \hline & Total-OFCG $P$ & Main-OFCG $P x$ & Orthogonal-OFCG $P y$ \\
\hline Span@3 dB & $105 \mathrm{GHz} / 21$ modes & $95 \mathrm{GHz} / 19$ modes & $55 \mathrm{GHz} / 11$ modes \\
Span@10 dB & $120 \mathrm{GHz} / 24$ modes & $115 \mathrm{GHz} / 23$ modes & $110 \mathrm{GHz} / 22$ modes \\
Flatness: f3 dB $=$ & $21 / 24=0.88$ & $19 / 23=0.83$ & $11 / 22=0.5$ \\
$3 \mathrm{~dB} / 10 \mathrm{~dB}$ & & & \\
Span@20 dB & $135 \mathrm{GHz} / 27$ modes & $135 \mathrm{GHz} / 27$ modes & $130 \mathrm{GHz} / 26$ modes \\
Flatness f10 dB $=$ & $24 / 27=0.89$ & $23 / 27=0.85$ & $22 / 26=0.85$ \\
$10 \mathrm{~dB} / 20 \mathrm{~dB}$ & & & \\
\hline \hline
\end{tabular}

$\mathrm{dBm})$. For this study, we have defined the comb flatness as the ratio of the $3 \mathrm{~dB}$ to the $10 \mathrm{~dB}$ optical span and the 10 to the $20 \mathrm{~dB}$ optical span. The closer these values are to 1 , the closer the comb is to exhibit a flat-top shape, more desirable for applications such as optical communications. The main results for this analysis of the OFCs generated are summarized in Table I. The total output of the VCSEL, $P$-OFCG that we show in Fig. 5(a) (upper trace) has 27 lines in the $20 \mathrm{~dB}$ span what corresponds to a bandwidth of $135 \mathrm{GHz}$ and a flatness of 0.88 and 0.89 respectively (see Table I). The same $20 \mathrm{~dB}$ span is measured in the comb with only the main polarization mode, the $P x$-OFCG shown in Fig. 5(b) (middle line) of 27 modes and $135 \mathrm{GHz}$. The 3 and $10 \mathrm{~dB}$ spans are 19 and 23 modes respectively so the flatness is 0.83 and 0.85 in each case. Finally, we see the orthogonal comb, $P y$-OFCG in Fig. 5(c) (lower line) which is slightly narrower with 26 lines that imply $130 \mathrm{GHz}$ in the $20 \mathrm{~dB}$ span. In this case the 3 and $10 \mathrm{~dB}$ spans are 11 and 22 modes and the flatness is 0.5 and 0.85 respectively. Then, the flatness of this comb is significantly worse than the one exhibited by $P$ and $P x$, and it has a different shape, shifted in frequency, as the initial secondary mode was.

Comparing these three combs we can distinguish each polarization sub-comb in the total one. The part of the total comb above the $20 \mathrm{~dB}$ line is mainly formed by the energy with the parallel polarization and we can see that their shapes match perfectly in this central part. However, if we focus on the right decay slope of the total comb (longer wavelengths around $1539.3 \mathrm{~nm}$ ) we observe that a small hip appears whose shape corresponds to the right part of the orthogonally polarized OFCG, the $P y$ OFCG which has much lower power. There we also see that the $P x$-OFCG is more symmetric than the total comb because these orthogonally polarized components are not present. If the optical power associated to the orthogonal mode could be enhanced, a total optical comb with wider span could be achieved with VCSEL-based OFCG schemes.

Special attention deserves the evolution of the $P y$-OFCG with the radiofrequency modulation power $P_{\mathrm{RF}}$, since this is the parameter that more clearly influences the formation of optical combs under GS regime. The map of such evolution was presented in Figs. 4, and in Fig. 6 we show the evolution of the shape of the $P y$ comb more in detail. When the RF modulation power is below $-10 \mathrm{dBm}$, the optical spectra of the orthogonally polarized light is formed by the secondary mode, with a peak power of $-50 \mathrm{dBm}$ and a residual emission coming from the main mode with a peak power of $-43 \mathrm{dBm}$. These two orthogonally polarized components of the output of the laser evolve as 


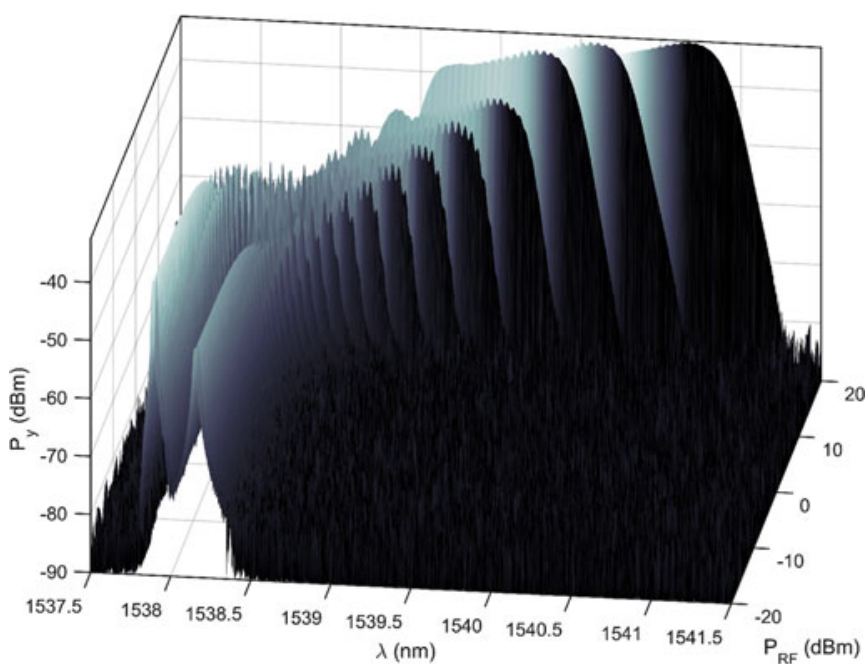

Fig. 6. Evolution of the optical comb with ortogonal polarization, $P y$ when $P_{\mathrm{RF}}$ increases. Initially, two separate comb-like structures are obsevable, that eventaully melt to produce the final ortogonal comb $P y$.

the GS regime is established. Initially, two separated comb-like spectral shapes appear associated to each of these components, being the one associated to the secondary mode the one whose peak power and optical span evolves faster. When $P_{\mathrm{RF}}$ is increased further, the two comb-like structures melt in a single optical comb with orthogonal polarization. After the study of the dynamics of the formation of this orthogonal comb, it is important to evaluate whether this two initial structures end up forming a coherent unique comb and how this $P y$ comb is related to the main $P x$ comb with parallel polarization. Results on this matter are presented in the next section.

In order to summarize this optical frequency comb generation section, we would like to remark the fundamental conclusions obtained: a) the three combs evolve similarly with the supply parameters and they all offer the best performance at $20{ }^{\circ} \mathrm{C}$ of VCSEL temperature, $11.5 \mathrm{~mA}$ of bias current, $5 \mathrm{GHz}$ and $16 \mathrm{dBm}$ as modulation signal; b) the power difference between the parallel and orthogonal mode respectively decreases significantly with the GS regime, from $43.1 \mathrm{~dB}$ of difference in the $\mathrm{CW}$ operation to $26 \mathrm{~dB}$ when the modulation in included; and c) both combs seems to be related to each other and they both generate the total comb. Further work is presented in the following section in order to extract whether the total comb is formed by two coherent combs with perpendicular polarizations or they are two independent signals and there is no phase relation between them.

\section{B. Coherence of the Modes of the Comb}

In this section we are paying attention to the coherence of the modes that form the combs. We evaluate this coherence by measuring the electrical beat tone signal at $f_{\mathrm{RF}}$ that is generated when directly detected in an ultrafast photodiode the signal of each of the combs under study: the ones associated to $P, P x$ and $P y$ signals. Hence, we evaluate mixing all the modes of

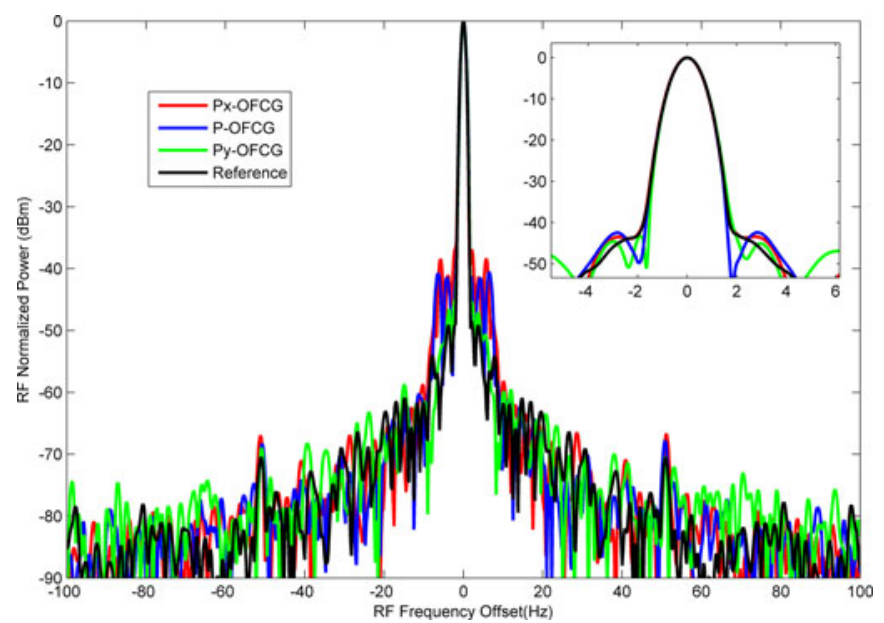

Fig. 7. RF Linewidth for the combs in Fig. 5: $P$-OFCG (blue line), $P x$-OFCG (red line), $P y$-OFCG (green line) and Reference (black line). In the inset we plot a zoom of these signals obtained with the lower Resolution Bandwidth available in the ESA used Therefore in all cases the linewidth is limited by the equipment capabilities. See text for more details.

each comb under study and analyse the electrical spectra and the linewidth of the line at $f_{\mathrm{RF}}$. An increase of the linewidth of this tone or a degradation of the noise present in the spectra is directly related to a decrease in the coherence between the modes and a degradation in the quality of the comb. In previous works, we have already seen the high coherence in the modes of the total comb generated with GS ( $P$-OFCG in this article) with a beat note signal with noise level equal the one in the reference signal (the CW source) [7] and how this result is also valid if we add other elements like Electro-Optical Modulators maintaining the initial GS regime in the VCSEL [8]. This implies that the use of a GS technique improves the coherence relation between the different modes in the final comb. Now we want to study if this coherence is maintained among these sub-combs with orthogonal polarization states. Moreover we also want to check with the electrical spectra, the phase coherence between both orthogonally polarized combs to test if they are two independent combs or they are somehow related to each other.

Thus, to evaluate the coherence of the modes in each subcomb we compare the linewidth of the reference signal (the RF CW source used to induce the GS regime) at $f_{\mathrm{RF}}$ and the linewidth of the beat tone signals at $f_{\mathrm{RF}}$ for each of the combs. In Fig. 7 we present the curves for the total comb $P$-OFCG (blue line), the main comb $P x$-OFCG (red line), the orthogonal comb $P y-O F C G$ (green line) and the reference signal which is the radiofrequency $\mathrm{CW}$ source at $5 \mathrm{GHz}$ (black line). In the figure we see that all the combs present the same decay slope which is slightly broader than the reference one at around $-40 \mathrm{~dB}$ from the carrier peak, where we find a broadening of the three combs in respect to the reference. In all these traces the floor is found at $-80 \mathrm{dBm}$. On the other hand, we show in the inset a zoom of the beat tone where we can see that the $3 \mathrm{~dB}$ linewidth is, for every signal, around $1 \mathrm{~Hz}$. This means that is in the equipment limit as the minimum resolution bandwidth is this value, $1 \mathrm{~Hz}$. 


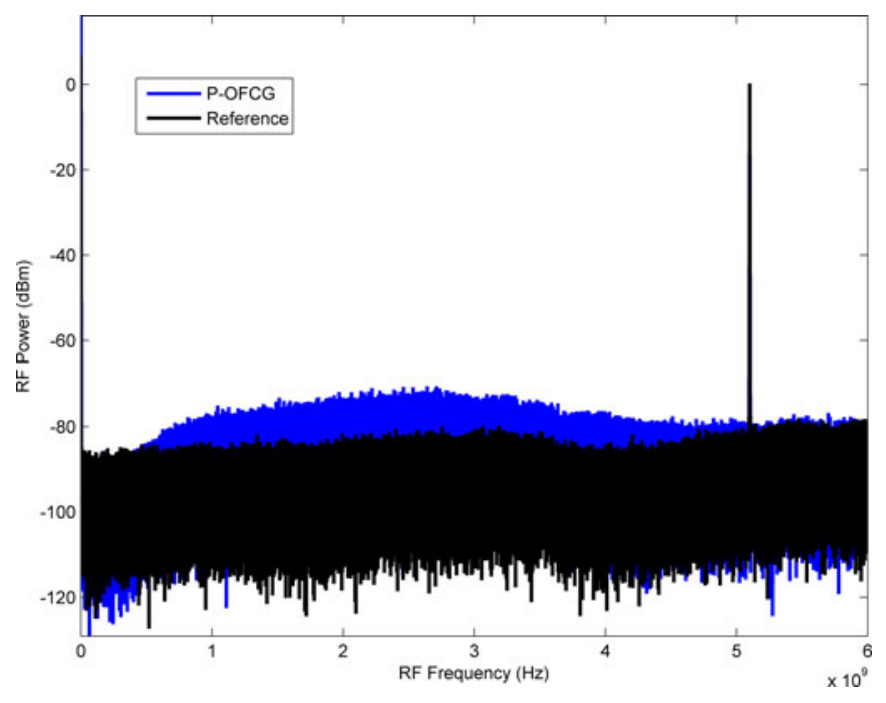

Fig. 8. RF Spectra for the total combs $P$-OFCG (blue line) and Reference (black line). We see that the noise level increases in the $P$-OFCG in respect to the reference curve but still is a remarkable low noise with the RF frequency as the only frequency component. This imply high correlation between the modes in the different combs. See text for more details.

With this figure we deduce that each comb has high coherence in their modes and we have high quality combs as the beat tone signal is always in the order of the reference equipment used for modulation.

On the other hand, we want to evaluate the coherence between modes that belong to different sub-combs. For this purpose, we compare the differences in the electrical spectra of each comb with higher span than the used in the previous image, from DC to some $\mathrm{MHz}$ above the RF injected frequency. We want to see if new frequency components appear in the RF spectra (different from $5 \mathrm{GHz}$ ) and then both combs are completely independent or not. In the latter case, the main and the orthogonal combs would be phase related and might be locked. In Fig. 8 we show the electrical spectra curve for the total comb P-OFCG (blue line) and the electrical spectrum of the reference signal, directly at the output of the CW source (black line).

The total comb curve presents a clear peak at the RF frequency of $5 \mathrm{GHz}$ and no other frequency components appear. This implies that both sub-combs, $P x$-OFCG and $P y$-OFCG, are phase correlated and not free running in respect to each other. Besides, the noise floor in the P-OFCG curve is close to the reference one and then the total comb is a signal with high coherence between all its modes as we were expecting taking into account our previous results [7]. This high coherence is driven by the GS regime because the polarization modes of a VCSEL are in CW operation anti-correlated regarding [10], [11].

\section{CONCLUSION}

In this work, we have presented a study of the polarization dynamics of VCSELs under GS regime, and how these polarizations contribute in the OFCGs with COTS components. OFCGs are interesting systems for many applications and LDs will allow these systems to become more efficient in cost and energy and more compact at the same time. Inside LDs technologies, VCSELs increment the impact in these three factors, they are easy to integrate and produces at the same time wider combs in comparison to other LDs technologies. GS modulation for generating these OFCGs is a versatile technique that yields to easy generation with tunable mode distance and no need of extra components in the set-up.

Analyzing the VCSEL-based GS-OFCG we observe that both polarization components form two different OFCGs, each of them with orthogonal polarization in respect to each other and they are correlated to each other. This correlation is a consequence of the GS regime as both polarization modes are, in CW operation, anti-correlated. Along this work, we have studied the quality of this OFCG in terms of optical span and flatness and also RF linewidth of the detected beat note of each comb and noise after being detected. For this purpose, here we have analyzed the VCSEL-based OFCG and the properties of the two orthogonally polarized combs that form this optical signal. First we have analyzed the optical spectra of the whole signal and the two different combs, each one with orthogonal polarizations. We have seen that they all evolve with the same pattern depending on the supply characteristics and they all have the best performance at $20{ }^{\circ} \mathrm{C}$ of VCSEL temperature, $11.5 \mathrm{~mA}$ of bias current, $5 \mathrm{GHz}$ of modulation frequency and $16 \mathrm{dBm}$ as modulation power. We have seen that one of the modes, with an orthogonal polarization, has much lower output power because of the birefringence of the material and a fabrication pinning process but it still produces an optical comb and in this comb the difference in power between both modes is reduced with GS regime compared to the power difference in CW operation. Apart of this optical study, we have presented a RF study where we have checked that each of the sub-combs present high correlation between their modes as the beat tone signal presents a linewidth comparable to the reference and in the limit of the equipment used for measure. We have also seen that both sub-combs are phase correlated to each other and not free running.

Future work will be developed in several lines: on one hand we are interested in repeat these measurements with VCSELs with less orthogonal mode suppression in order to directly compare both sub-combs and go further in the analysis of the polarization components of VCSELs and their influence in comb generation. On the other hand, we would like to check if we can obtain, with Optical Injection Locking, two correlated and completely locked combs with similar properties in which we can play with the polarization with techniques like the ones observed in [17]. We will also work in order to increase the optical power in the orthogonal comb by including Optical Injection Locking of each one of the combs, the Px-OFCG and the $P y$-OFCG respectively and how this affects the total comb, $P$-OFCG. Another future line consists in changing the birefringence of the VCSEL sample, varying the distance in the central wavelength of both sub-combs and increasing also the modulation frequency range available. Experiments like this have been previously done in works like [18]. 
To conclude, our VCSEL-based GS-OFCG offers, in the few $\mathrm{GHz}$ repetition frequency rates, dual-polarization combs with tunable distance and high correlation in their modes in an easy, energy efficient and easy to integrate system, with applications in ultrafast laser dynamics studies or in polarization-division multiplexing optical communications. All these features make VCSEL-based GS-OFCGs promising systems to produce low cost and high quality OFCGrs which is a tendency nowadays. However, the optical span and the flatness are still limiting factors in comparison to other non LDs comb generation techniques. Our work continues in order to improve the generated signal and more deeply understand the dynamics of our LD source.

\section{ACKNOWLEDGMENT}

The authors would like to thank VERTILAS for providing the VCSELs.

\section{REFERENCES}

[1] F. Zhu, T. Mohamed, J. Strohaber, A. A. Kolomenskii, T. Udem, and H. A. Schuessler, "Real-time dual frequency comb spectroscopy in the near infrared," Appl. Phys. Lett., vol. 102, no. 12, p. 121116, 2013.

[2] R. Michalzik, VCSELs, vol. 166. Berlin, Germany: Springer, 2013.

[3] A. R. Criado, C. De Dios, G. H. Döhler, S. Preu, S. Malzer, S. Bauerschmidt, H. Lu, A. C. Gossard, and P. Acedo, "Ultra-narrow linewidth CW sub-THz generation using GS based OFCG and n-i-pn-i-p superlattice photomixers," Electron. Lett., vol. 48, pp. 1425-1426, 2012.

[4] R. Wu, C. M. Long, D. E. Leaird, and A. M. Weiner, "Directly generated Gaussian-shaped optical frequency comb for microwave photonic filtering and picosecond pulse generation," IEEE Photon. Technol. Lett., vol. 24, no. 17 , pp. $1484-1486$, Sep. 2012.

[5] H. E. Li and K. Iga, Vertical-Cavity Surface-Emitting Laser Devices, vol. 6. Berlin, Germany: Springer, 2003.

[6] K. Iga, "Surface-emitting laser-its birth and generation of new optoelectronics field," IEEE J. Sel. Topics Quantum Electron., vol. 6, no. 6, pp. 1201-1215, Nov. 2000.

[7] A. R. Criado, C. De Dios, E. Prior, M. Ortsiefer, P. Meissner, and P. Acedo, "VCSEL-based optical frequency combs: Toward efficient single-device comb generation," IEEE Photon. Technol. Lett., vol. 25, no. 20, pp. 1981-1984, Oct. 2013.

[8] E. Prior, C. De Dios, A. R. Criado, M. Ortsiefer, P. Meissner, and P. Acedo, "Experimental study of VCSEL-based optical frequency comb generators," IEEE Photon. Technol. Lett., vol. 26, no. 21, pp. 2118-2121, Nov. 2014.

[9] J. Danckaert, B. Nagler, J. Albert, K. Panajotov, I. Veretennicoff, and T. Erneux, "Minimal rate equations describing polarization switching in vertical-cavity surface-emitting lasers," Opt. Commun., vol. 201, nos. 1-3, pp. 129-137, Jan. 2002.

[10] J.-L. Vey, C. Degen, K. Auen, and W. Elsäßer, "Quantum noise and polarization properties of vertical-cavity surface-emitting lasers," Phys. Rev. A, vol. 60, no. 4, pp. 3284-3295, Oct. 1999.

[11] D. Shelly, T. Garrison, M. K. Beck, and D. Christensen, "Polarization correlations in pulsed, vertical cavity, surface-emitting lasers," Opt. Exp. vol. 7, no. 7, p. 249 , Sep. 2000

[12] M. Brunel, J. Thévenin, and M. Vallet, "Dual-polarization frequency comb from a diode-pumped solid-state laser," presented at the Proc. : 2013Conf Lasers Electro-Opt., San Jose, CA, USA, 2013, Paper CF1G.5.

[13] A. D. Shiner, M. Reimer, A. Borowiec, S. O. Gharan, J. Gaudette, P. Mehta D. Charlton, K. Roberts, and M. O'Sullivan, "Demonstration of an 8dimensional modulation format with reduced inter-channel nonlinearities in a polarization multiplexed coherent system," Opt. Exp., vol. 22, no. 17 pp. 20366-20374, Aug. 2014.

[14] J. Albert, G. Van Der Sande, B. Nagler, K. Panajotov, I. Veretennicoff, J. Danckaert, and T. Erneux, "The effects of nonlinear gain on the stability of semi-degenerate two-mode semiconductor lasers: A case study on VCSELs," Opt. Commun., vol. 248, nos. 4-6, pp. 527-534, Apr. 2005.
[15] A. R. Criado, C. De Dios, E. Prior, G. H. Dohler, S. Preu, S. Malzer, H. Lu, A. C. Gossard, and P. Acedo, "Continuous-wave sub-THz photonic generation with ultra-narrow linewidth, ultra-high resolution, full frequency range coverage and high long-term frequency stability," IEEE Trans. Terahertz Sci. Technol., vol. 3, no. 4, pp. 461-471, Jul. 2013.

[16] P. M. Anandarajah, R. Maher, Y. Q. Xu, S. Latkowski, J. O'Carroll, S. G. Murdoch, R. Phelan, J. O'Gorman, and L. P. Barry, "Generation of coherent multicarrier signals by gain switching of discrete mode lasers," IEEE Photon. J., vol. 3, no. 1, pp. 112-122, Feb. 2011.

[17] A. Quirce, P. Perez, H. Lin, A. Valle, L. Pesquera, K. Panajotov, and H. Thienpont, "Polarization switching regions of optically injected longwavelength VCSELs," IEEE J. Quantum Electron., vol. 50, no. 11, pp. 921-928, Nov. 2014.

[18] K. Panajotov, B. Nagler, G. Verschaffelt, A. Georgievski, H. Thienpont, J. Danckaert, and I. Veretennicoff, "Impact of in-plane anisotropic strain on the polarization behavior of vertical-cavity surface-emitting lasers," Appl. Phys. Lett., vol. 77, no. 11, p. 1590, Sep. 2000.

Estefanía Prior received the B.Sc. and M.Sc. degrees in telecommunication engineering from the Universidad Carlos III de Madrid, Madrid, Spain, in 2012, and the M.Res. in electronics engineering in 2014. She is currently working toward the Ph.D. degree at the Carlos III University of Madrid. In 2012, she was involved in research and development tasks related to microwave photonics and radio over fiber, in both transmission and receiver systems. Since 2013, her work has been focusing on pulsed operation (gain switching) on semiconductor lasers and, more specifically, on vertical-cavity surface-emitting lasers for optical frequency comb generation.

Cristina de Dios received the M.Sc. degree in applied physics and electronics from the Universidad Complutense of Madrid, Madrid, Spain. Then, she joined the private sector as a Technical Consultant for Hewlett-Packard. Since 2002, she has been a Member of the Optoelectronics and Laser Technology Group, Universidad Carlos III de Madrid, Madrid, where she received the M.Res. in 2004 and the Doctorate degree in 2010 for her work in ultrafast pulsed diode lasers and nonlinear pulse compression. She is currently an Assistant Professor at the Electronics Technology Department, Universidad Carlos III de Madrid. She is also cofounder of the spin-off company Luzwavelabs (www.luzwavelabs.com) that provides hardware solutions for generation and detection in the $\mathrm{THz}$ range using photonic techniques. Her research interests include high-speed optical communications, pulsed semiconductor laser sources, nonlinear optical phenomena, and subterahertz and millimeter wave photonic signal synthesis and detection and optical frequency comb generation.

Markus Ortsiefer received the University degree in physics and the doctoral degree from the Technical University of Munich, Munich, Denmark, in 1997 and 2001, respectively. During his Ph.D. work, he was involved in research on InPbased lasers and related materials. He is the Co-Founder of VERTILAS GmbH, Garching, Germany, where he was the Managing Director from 2001 to 2003, and since 2003, he has been the Chief Technical Officer. He is responsible for the company's production and research activities. He has authored or coauthored more than 130 publications in scientific journals, conference proceedings, and books and filed several patents on optoelectronic devices. He is a Member of the German Physical Society. 
Peter Meissner (M'94) was born in Adelsdorf, Germany, on November 1943. He received the Dip.-Ing. and Dr.-Ing. degrees in electrical engineering from the Technical University, Berlin, Germany, in 1971 and 1977, respectively. In 1971, he was with the Heinrich Hertz Institut für Nachrichtentechnik, Berlin, where he was involved in the field of navigation systems and adaptive echo control. From 1985 to 1994, he was involved in the field of optical communications, where his main interests were theory of semiconductor lasers, coherent optical communications, and wavelength division multiplexing (WDM) systems and networks. Since 1995, he has been a Professor of optical communications with the Technische Universität Darmstadt, Darmstadt, Germany. His main research topics are tunable micromachined components for WDM systems and high-speed optical communications, polarization mode dispersion compensation techniques, and optical terahertz generation.
Pablo Acedo (M'00) received the Bachelor's degree in telecommunication engineering from the Universidad Politécnica de Madrid, Madrid, Spain, in 1993 , and the Doctorate (Hons.) degree from the Universidad Carlos III de Madrid, Madrid, in 2000 for his work on heterodyne two color laser interferometry for fusion plasma diagnostics. His doctoral work included the development of the first two color laser system based on Mid-IR sources for a Stellarator Fusion Device (Stellarator TJ-II, Laboratorio Nacional de Fusión, CIEMAT, Madrid) and the first two-color Nd:YAG system for a Fusion Device (Tokamak C-Mod Plasma Science and Fusion Centre, Massachusetts Institute of TechnologyMIT), the later during several doctoral visits to MIT during 1996-1999.

In 2002, he became an Assistant Professor at the Universidad Carlos III de Madrid, where he continued with the development of scientific instrumentation systems for fusion plasma diagnostics and biomedical applications, leading national projects and contracts on these fields. Also, since his incorporation to the UC3M, he has been involved in research with the Optoelectronics and Laser Technology Group, participating in different national and EU funded research projects (Fp4, Fp5, Fp6, and FP7) on semiconductor laser devices and photonic architectures for microwave, millimeter-wave, and terahertz generation and processing. Starting 2009, his research interests in this line focused on the development of multimode laser sources (optical frequency combs) and their applications in photonic signal synthesis with the development of several architectures for the generation, detection and processing of terahertz signals, leading several research projects and contracts in this field. He has also been very active in technology transfer through R\&D contracts with different companies (INDRA Sistemas S.A., AIRBUS Defence and Space) as well as with the creation of the spin-off company Luzwavelabs (www.luzwavelabs.com) that provides hardware solutions for generation and detection in the terahertz range using photonic techniques. Nowadays, his research interests still involve the development of optical frequency combs and their use in fields such as bioengineering, environmental applications, and nondestructive testing. He has published more than 80 contributions in journals and international conferences, including invited conferences and seminars. 